\title{
Averaged null energy condition in spacetimes with boundaries
}

\author{
Christopher J. Fewster* \\ Department of Mathematics, University of York, \\ Heslington, York, YO10 5DD, U.K. \\ Ken D. Olum \\ Institute of Cosmology, Department of Physics and Astronomy, \\ Tufts University, Medford, MA 02155 \\ Michael J. Pfenning \\ Department of Physics, United States Military Academy, West Point, NY 10996
}

(Dated: 28 November 2006)

\begin{abstract}
The Averaged Null Energy Condition (ANEC) requires that the average along a complete null geodesic of the projection of the stress-energy tensor onto the geodesic tangent vector can never be negative. It is sufficient to rule out many exotic phenomena in general relativity. Subject to certain conditions, we show that the ANEC can never be violated by a quantized minimally coupled free scalar field along a complete null geodesic surrounded by a tubular neighborhood in which the geometry is flat and whose intrinsic causal structure coincides with that induced from the full spacetime. In particular, the ANEC holds in flat space with boundaries, as in the Casimir effect, for geodesics which stay a finite distance away from the boundary.
\end{abstract}

PACS numbers: $03.70 .+\mathrm{k} 04.20 . \mathrm{Gz}$

*Electronic address: cjf3@york.ac.uk

$\dagger$ Electronic address: kdo@cosmos.phy.tufts.edu

${ }^{\ddagger}$ Electronic address: Michael.Pfenning@usma.edu 


\section{INTRODUCTION}

General relativity alone gives no restriction on the geometry or topology of spacetime. Simply by solving Einstein's equation, $G_{a b}=\kappa T_{a b}$, in reverse, one may easily determine the stress-energy tensor $T_{a b}$ needed to sustain any desired spacetime. Thus the only possibility for restricting exotic phenomena such as superluminal travel [1], traversable wormholes [2], or the creation of time machines [3, 4] is to have energy conditions that restrict $T_{a b}$. Sufficient to rule out the exotic phenomena above and to prove classical singularity theorems is the null energy condition (NEC), which requires that $T_{a b}(x) V^{a} V^{b} \geq 0$ for all spacetime points $x$ and null vectors $V$.

Unfortunately, the NEC is violated when we introduce quantum fields; an example is the original Casimir effect [5]. For any $x$ between the (infinite) plates and any vector $V$ which is not parallel to the plates, $\left\langle T_{a b}(x) V^{a} V^{b}\right\rangle$ will be negative in the ground state.

A weaker condition is the averaged null energy condition (ANEC), which requires only that

$$
\int_{\zeta} d v T_{a b} k^{a} k^{b} \geq 0
$$

where $\zeta(v)$ is a complete affinely parameterized null geodesic with tangent vector $k$. This condition is not expected to be violated by the Casimir plate system, because geodesics parallel to the plates do not violate the NEC, while any other geodesic eventually intersects the plate material, where it picks up a positive contribution to the integral. The ANEC is sufficient to rule out traversable wormholes and the construction of time machines. Variations of it can also be used to prove singularity theorems [6, 7, 8] and to rule out superluminal travel $^{1}[1]$.

Various proofs of the ANEC for quantum matter are known. In $n$-dimensional Minkowski space, the ANEC was proved for the scalar field with arbitrary curvature coupling for a dense set of states in the Fock space of the Minkowski vacuum [9]; namely, all finite linear combinations of finite particle-number states of bounded momentum. Folacci has posited a similar argument for electromagnetism in four dimensions [10]. One may also obtain the ANEC as a limiting case of the averaged weak energy condition (AWEC) ${ }^{2}$ [which holds in Minkowski space as a consequence of Quantum Energy Inequalities] [11] by considering the null geodesic as a limit of timelike curves; the relevant class of states is not so clearly defined here and quite strong conditions are required at infinity to make this work (see [12] for some comments in this direction). A more general and quite technical argument [13], using techniques of algebraic quantum field theory, establishes the ANEC in four-dimensional Minkowski space for a restricted class of Hadamard states of the minimally coupled scalar field. In particular, this goes beyond the states contained in the usual Fock space.

In two dimensions more can be said: in Minkowski space one may prove the ANEC for general (interacting) quantum fields with mass [14], or for unitary, positive energy conformal fields [15]; precise classes of states for which these results hold are delineated in each case. In general globally hyperbolic two-dimensional spacetimes, [13] established the ANEC on complete achronal null geodesics for the minimally coupled free scalar field. This holds for arbitrary Hadamard states in the massless case, and a restricted class of Hadamard states

\footnotetext{
${ }^{1}$ The condition used in [1] is in fact the NEC, but one can see that is sufficient to have the ANEC with the average over the path to be traveled.

${ }^{2}$ The AWEC requires that (1) holds with $\zeta$ replaced by a complete timelike geodesic.
} 
for nonzero mass; in neither case was there any requirement that the states should be vectors in a Fock space representation. The achronal condition on the geodesic may be dropped [16] at the expense of passing to a "difference ANEC".

It is known that the ANEC may be violated in general curved spacetimes (e.g., see [17]) and in semi-classical quantum gravity [18]. Indeed, wormhole solutions have been found in which the spacetime curvature leads to the ANEC violation that supports the wormhole [19, 20, 21], but these involve Planck-scale structures, and thus should require a full quantum gravitational treatment. If one avoids physics at the Planck scale, exotic phenomena can exist only with a very large separation of scales [22, 23]. The ANEC is also violated for the ground state of the Klein-Gordon field in flat space with a periodic identification in one spatial direction along any null geodesic which winds around the compact direction. We will return to this case, which is essentially the Casimir system without the plates, in Sec. IV.

The above results all refer to globally hyperbolic spacetimes, which are by definition boundaryless. Several attempts [24, 25, 26, 27, 28] have been made to violate the ANEC with specific systems of free fields with boundaries, but these have been unsuccessful. Ref. [25] conjectured that the ANEC is obeyed for all geodesics passing outside a localized potential in flat space.

We will prove here a stronger version of the conjecture of [25]. Namely, we will show that distant boundaries or potentials do not affect the ANEC, and that distant spacetime curvature likewise has no effect if it does not change the causal structure near the geodesic. By "distant" here we mean simply that the geodesic must not approach arbitrarily close to such places.

The central idea is that the presence of distant boundaries can only be detected if one can send a signal to the boundary and receive a signal in return. Otherwise one cannot determine whether one is making measurements in spacetime with boundaries or merely observing an unusual state on an unbounded spacetime. But no signal can leave a null geodesic in flat space and later return to it (neglecting, for the moment, situations with nontrivial topology). Thus for measurements only on the geodesic, the boundaries cannot be observed, and such a measurement must correspond to a possible measurement on the geodesic in Minkowski space. But the ANEC is obeyed in Minkowski space, so we expect the ANEC to be obeyed even if there are boundaries.

At a more technical level, the key ingredient is the Quantum Null Energy Inequality (QNEI) proved in [12], which places bounds on weighted averages of the null-contracted stress-energy tensor along timelike curves, and the covariance properties of this bound (and other related Quantum Energy Inequalities (QEIs)) as discussed in [29]. We refer the reader to [29] and references therein for general background on QEIs.

We will not be able to prove the ANEC without additional assumptions (which bridge the gap between the averages considered in [12] and the ANEC integral), but we will prove that there cannot be a timelike-separated family of geodesics on which Eq. (1) converges uniformly to negative values. To have some practical effect, one needs not merely a single geodesic on which the ANEC is violated but some finite region of violation. Furthermore, one expects that infinitesimally separated geodesics violate ANEC in the same way, so that the convergence is uniform. Thus we feel that ruling out uniform ANEC violation is sufficient for all practical purposes.

The paper is structured as follows. In Sec. II we rigorously state our premises and prove the conclusion. In Sec. III we give a variant argument in which we assume uniform continuity of $T_{a b}$ and then can prove the ANEC on a single geodesic. After discussing the example of 
cylindrical spacetime in Sec. IV], we conclude in Sec. V].

\section{MAIN RESULT}

\section{A. Geometrical assumptions}

Let $(N, \boldsymbol{g})$ be a spacetime composed of a four-dimensional smooth manifold $N$ (possibly with boundary) equipped with a Lorentzian metric $\boldsymbol{g}$ and choices of orientation and timeorientation. We do not assume that $(N, \boldsymbol{g})$ is globally hyperbolic, although we do assume that strong causality holds on the interior of $N$ and that the spacetime supports a suitable quantum field theory, as we explain in more detail below.

Our aim is to prove the ANEC along any complete null geodesic $\zeta$ in $N$ which is sufficiently isolated from the boundaries and regions of curvature. More precisely, we assume that the Riemann tensor vanishes everywhere in a simply-connected open neighborhood $N^{\prime}$ of $\zeta$ that has no intersection with the boundary of $N$, and further that

$$
J^{+}(p, N) \cap N^{\prime}=J^{+}\left(p, N^{\prime}\right) \quad \text { for all } p \in N^{\prime},
$$

i.e., regions outside $N^{\prime}$ have no effect on the causal structure inside $N^{\prime}$. (The causal future $J^{+}$can be replaced, equivalently, by the causal past $J^{-}$in (2).)

Given these assumptions, we can define coordinates $(t, x, y, z)$ on $N^{\prime}$ such that $\zeta$ may be affinely parameterized as $\zeta(\lambda)=(\lambda, \lambda, 0,0)$, and in which the metric takes the Minkowski form $d s^{2}=d t^{2}-d x^{2}-d y^{2}-d z^{2}$ on $N^{\prime}$. Without loss of generality it may also be assumed that the tetrad $(\partial / \partial t, \partial / \partial x, \partial / \partial y, \partial / \partial z)$ is oriented and time-oriented, so our coordinates define a (time-)orientation preserving isometry $\psi: N^{\prime} \rightarrow M^{\prime}$, where $M^{\prime}$ is a region of Minkowski space $M$. form

Finally, we assume that $N^{\prime}$ is large enough to contain a tubular neighborhood of $\zeta$ of the

$$
N_{r}^{\prime}=\left\{(t, x, y, z) \mid y^{2}+z^{2}+(x-t)^{2}<r^{2}\right\}
$$

for some $r>0$. Thus $N^{\prime}$ is not permitted to "taper off" at infinity, thereby (for example) excluding situations where $\zeta$ is asymptotic to the boundary of $N$ at large values of the affine parameter, or where it passes through a sequence of apertures of diminishing radius.

Note that condition (2) can only hold if the null geodesic $\zeta$ is achronal. For suppose there are points $p, q$ on $\zeta$ with $q \in I^{+}(p, N)$. Then $q$ is an interior point of $J^{+}(p, N) \cap N^{\prime}$, but a boundary point of $J^{+}\left(p, N^{\prime}\right)$, so these sets cannot be equal.

\section{B. Assumptions on the quantum field theory}

We study the minimally coupled scalar field of mass $m \geq 0$ on $(N, \boldsymbol{g})$. As we have not assumed that $(N, \boldsymbol{g})$ is globally hyperbolic, we must make some further assumptions about the existence of a reasonable quantization of the theory on this spacetime. The guiding principle, motivated by the analysis of [30], is that the theory should coincide with the usual quantization on open globally hyperbolic subsets ${ }^{3}$ of the interior of $(N, \boldsymbol{g})$. Here, a subset

\footnotetext{
${ }^{3}$ In [29] these were referred to as c.e.g.h.s. regions.
} 
$U$ of $(N, \boldsymbol{g})$ is globally hyperbolic if strong causality holds on $U$ and $J^{+}(p, N) \cap J^{-}(q, N)$ is a compact subset of $U$ for all $p, q \in U$ (see Sec. 6.6 of [31]). In particular, if $U$ is also open, it may be regarded as a globally hyperbolic spacetime in its own right, but our condition also ensures that the intrinsic causal structure of $\left(U,\left.\boldsymbol{g}\right|_{U}\right)$ coincides with that induced from $(N, \boldsymbol{g})$. Thus, for example, 'commutation at spacelike separation' has the same meaning in the two spacetimes. As we have assumed that strong causality holds at each point of the interior of $N$, every such point has an open globally hyperbolic subset as a neighborhood.

We also restrict attention to states which restrict to open globally hyperbolic subsets of the interior of $N$ as Hadamard states. In particular, these conditions are met when $(N, \boldsymbol{g})$ is globally hyperbolic and we use the usual quantization, with the states of interest required to be Hadamard on the whole of $(N, \boldsymbol{g})$, but there are more general situations where they hold (see [32] for more details and references). These conditions are sufficient for the renormalized stress-energy tensor to be defined according to the usual point-splitting prescription. For simplicity, we will refer to our states as Hadamard, although they correspond to a generalization of the usual concept. The conditions given here would, we believe, be satisfied in any reasonable quantum field theory on $(N, \boldsymbol{g})$; we do not claim that they are sufficient conditions to guarantee the existence of such a theory.

The geometrical conditions on $\zeta$ and its neighborhood $N^{\prime}$ permit us to identify a useful class of open globally hyperbolic subsets of $(N, \boldsymbol{g})$ : let $p, q \in N^{\prime}$ and suppose that the Minkowski space 'double cone' $J^{+}(\psi(p), M) \cap J^{-}(\psi(q), M)$ is contained within $M^{\prime}$. Then the open set

$$
D_{p, q}=I^{+}\left(p, N^{\prime}\right) \cap I^{-}\left(q, N^{\prime}\right),
$$

is a globally hyperbolic subset of $(N, \boldsymbol{g})$. To see this, first note that strong causality holds on $D_{p, q}$ because it holds on the interior of $N$. Now choose $r, s \in D_{p, q}$ and consider $K=$ $J^{+}(r, N) \cap J^{-}(s, N)$. By property (2) [and the equivalent version for the causal past] the intersection $K \cap N^{\prime}$ is simply $J^{+}\left(r, N^{\prime}\right) \cap J^{-}\left(s, N^{\prime}\right)$, which is a subset of $D_{p, q} \cdot$ Thus $K$ is covered by the disjoint closed sets $\bar{D}_{p, q}$ and $N \backslash N^{\prime}$; since it is connected, it must in fact be contained entirely in $\bar{D}_{p, q}$. Thus $K=J^{+}\left(r, N^{\prime}\right) \cap J^{-}\left(s, N^{\prime}\right)$ and is therefore compact, because the corresponding Minkowski double cone is. Hence $D_{p, q}$ is an open globally hyperbolic subset of the interior of $(N, \boldsymbol{g})$.

The main technical tool we will use to prove the ANEC is a QNEI established in [12] (Thrm III.1) which showed that the quantity

$$
\int_{\gamma} d \tau\left\langle T_{a b} k^{a} k^{b}\right\rangle_{\omega}(\gamma(\tau)) g(\tau)^{2}
$$

is bounded from below as $\omega$ varies over the class of Hadamard states, where $\gamma$ is a smooth future-directed timelike curve parameterized by proper time $\tau \in I$, for $I$ an open interval of the real line, ${ }^{5} k$ is a smooth null vector field defined on a tubular neighborhood of $\gamma$ and $g$ is any smooth, real-valued function compactly supported in $I$. We must also require that $\gamma$ may be enclosed in an open globally hyperbolic subset of $(N, \boldsymbol{g})$, because the result of [12] was proved in the globally hyperbolic setting.

In general the lower bound given in [12] is exceedingly difficult to calculate for a general worldline in a generic spacetime (it depends on the two-point function of a reference

\footnotetext{
${ }^{4}$ We use the facts that $J^{+}\left(r, N^{\prime}\right) \subset I^{+}\left(p, N^{\prime}\right)$ and $J^{-}\left(s, N^{\prime}\right) \subset I^{-}\left(q, N^{\prime}\right)$.

${ }^{5}$ In [12] $I$ was taken to be the whole line, but the difference is inessential.
} 
Hadamard state). However, the bound simplifies considerably if $\gamma$ may be enclosed in an open globally hyperbolic subset $N^{\prime}$ of $(N, \boldsymbol{g})$ which is isometric to an open globally hyperbolic subset $M^{\prime}$ of Minkowski space in such a way that the (time-)orientation induced on $M^{\prime}$ by the isometry coincides with the usual (time-)orientation of Minkowksi space. Under these circumstances the QNEI bound reduces to the form it takes in Minkowski space (see Thrm. II.6 and Cor. II.4 of [29], which establish the required covariance property) and this has a simple closed form for the case where $\gamma$ is geodesic and $k^{a}$ is covariantly constant near $\gamma$. These bounds will be employed below for regions of the $D_{p, q}$ form mentioned above. It is also worth noting that [12] also showed that we cannot expect a quantum energy inequality to hold for averaging along a null geodesic.

\section{The ANEC}

We may now state our main result, assuming that the spacetime $(N, \boldsymbol{g})$, the complete null geodesic $\zeta$ and its neighborhood $N^{\prime}$ obey the geometrical conditions of Sect. IIA, and that $(N, \boldsymbol{g})$ admits a quantized real scalar field satisfying the conditions of Sect. IIB.

Define $\Phi_{0}(\lambda, t)=(t+\lambda, \lambda, 0,0)$, so that $\zeta(\lambda)=\Phi_{0}(\lambda, 0)$, and consider the ANEC integral,

$$
A(t)=\int_{-\infty}^{\infty} d \lambda T_{\omega}\left(\Phi_{0}(\lambda, t)\right)
$$

where $T_{\omega}(q)=\left\langle T_{a b}(q) k^{a} k^{b}\right\rangle_{\omega}$ is the renormalized expectation value of the stress-energy tensor $T_{a b}$, in Hadamard state $\omega$, at the point $q$ projected onto the null tangent vector $k=\partial \Phi_{0} / \partial \lambda$. Our main result is the following.

Theorem II.1. If $\omega$ is any Hadamard state then it is impossible for the integral in Eq. (6) to converge uniformly to negative values in any interval of $t$ containing 0 . That is, ANEC cannot be uniformly violated near $\zeta$.

We remark that, because $T_{\omega}(q)$ is continuous in $q, A(t)$ is also continuous in any interval of $t$ for which the integral in Eq. (6) converges uniformly. So an equivalent statement is that the integral cannot converge uniformly in an interval of $t$ containing 0 and have $A(0)<0$. Before giving the proof of Theorem II.1, we state an immediate consequence.

Corollary II.2. If $\left\langle T_{a b}\right\rangle_{\omega}$ is stationary with respect to $t$ in $N^{\prime}$ and the integral in Eq. (6) converges for $t=0$, then $A(0) \geq 0$, i,e., the ANEC holds on $\zeta$ in state $\omega$.

In order to prove Theorem II.1 we first introduce some families of timelike curves. Given any velocity $v$ with corresponding boost $\gamma=1 / \sqrt{1-v^{2}}$, define a family of timelike geodesic segments

$$
\Phi_{v}(\eta, \tau)=(\eta+\gamma \tau, \eta+\gamma v \tau, 0,0)
$$

parameterized by proper time $\tau \in\left(-\tau_{0}, \tau_{0}\right)$ and labeled by $\eta \in\left(-\eta_{0}, \eta_{0}\right)$. The choice of $v$, $\tau_{0}$ and $\eta_{0}$ will be discussed below. Now consider the set

$$
C_{v, \eta}=I^{+}\left(\Phi_{v}\left(\eta,-\tau_{0}\right), N^{\prime}\right) \cap I^{-}\left(\Phi_{v}\left(\eta, \tau_{0}\right), N^{\prime}\right) .
$$

Providing that $\tau_{0}<r$, where $N_{r}^{\prime}$ is of the form Eq. (3)), it is easy to show that the Minkowski space boosted double cone $J^{+}\left(\psi\left(\Phi_{v}\left(\eta,-\tau_{0}\right)\right), M\right) \cap J^{-}\left(\psi\left(\Phi_{v}\left(\eta, \tau_{0}\right)\right), M\right)$ is contained within the region $\psi\left(N_{r}^{\prime}\right) \subset M^{\prime}$; thus $C_{v, \eta}$ belongs to the family of open globally hyperbolic subsets of $(N, \boldsymbol{g})$ discussed in Sec. IIB, 
Accordingly, the discussion above entails that the QNEI of [12] may be applied to the segment $\tau \mapsto \Phi_{v}(\eta, \tau)$ as if it were in Minkowski space. Let $f(a)$ be any smooth real-valued sampling function with support only for $|a|<1$ and normalization

$$
\int f(a)^{2} d a=1
$$

Then, from Eq. (3.10) of [12] [applied to $g(\tau)=f\left(\tau / \tau_{0}\right)$ ] we have

$$
\int_{-\infty}^{\infty} d \tau T_{\omega}\left(\Phi_{v}(\eta, \tau)\right) f\left(\tau / \tau_{0}\right)^{2} \geq-\frac{\left(v_{a} k^{a}\right)^{2}}{12 \pi^{2} \tau_{0}^{4}} \int_{-\infty}^{\infty} d \tau f^{\prime \prime}\left(\tau / \tau_{0}\right)^{2}
$$

where the timelike tangent vector $v=\partial \Phi_{v} / \partial \tau$, null vector $k=\partial \Phi_{v} / \partial \eta$, and the prime denotes the derivative of the function with respect to its argument. ${ }^{6}$ Thus $\left(v_{a} k^{a}\right)^{2}=(1-$ $v) /(1+v)<1 / \gamma^{2}$, and so

$$
\int_{-\infty}^{\infty} d \tau T_{\omega}\left(\Phi_{v}(\eta, \tau)\right) f\left(\tau / \tau_{0}\right)^{2} \geq-\frac{F}{12 \pi^{2} \gamma^{2} \tau_{0}^{3}}
$$

where

$$
F=\int f^{\prime \prime}(a)^{2} d a
$$

is a manifestly positive constant.

The points $\Phi_{v}(\eta, \tau)$ with $-\tau_{0}<\tau<\tau_{0}$ and $-\eta_{0}<\eta<\eta_{0}$ form a parallelogram, as shown in Fig. 1. We can integrate over this parallelogram,

$$
\int_{-\eta_{0}}^{\eta_{0}} d \eta \int_{-\tau_{0}}^{\tau_{0}} d \tau T_{\omega}\left(\Phi_{v}(\eta, \tau)\right) f\left(\tau / \tau_{0}\right)^{2} \geq-\frac{\eta_{0} F}{6 \pi^{2} \gamma^{2} \tau_{0}^{3}}
$$

By holding $\tau_{0}$ fixed and increasing $\gamma^{2}$ faster than $\eta_{0}$, we can make the right hand side arbitrarily close to 0 .

We are now in a position to prove Theorem II.1. Suppose that the integral in Eq. (6) converges uniformly to negative values in an interval of $t$ containing 0 . Thus, there are some positive numbers $A$ and $t_{0}$ (without loss of generality, we take $t_{0}<r$ ) such that

$$
A(t)<-A \quad \text { for all } t \in\left(-t_{0}, t_{0}\right) .
$$

The parallelogram $\Phi_{v}(\eta, \tau)$ can also be considered as a set of null geodesics parameterized by $\eta$ and labeled by $\tau$. Then $\Phi_{v}(\eta, \tau)=\Phi_{0}(\lambda, t)$ with the correspondence

$$
\begin{aligned}
t & =(1-v) \gamma \tau \\
\lambda & =\eta+\gamma v \tau
\end{aligned}
$$

so our parallelogram is $\Phi_{0}(\lambda, t)$ with $t \in\left(-t_{1}, t_{1}\right)$ and $\lambda \in\left(\lambda_{-}, \lambda_{+}\right)$where $t_{1}=(1-v) \gamma \tau_{0}=$ $\tau_{0} /(\gamma(1+v))$ and $\lambda_{ \pm}= \pm \eta_{0}+t v /(1-v)$.

\footnotetext{
$\overline{6}$ This bound was derived in [12] for the massless field, but also applies in the case $m>0$.
} 


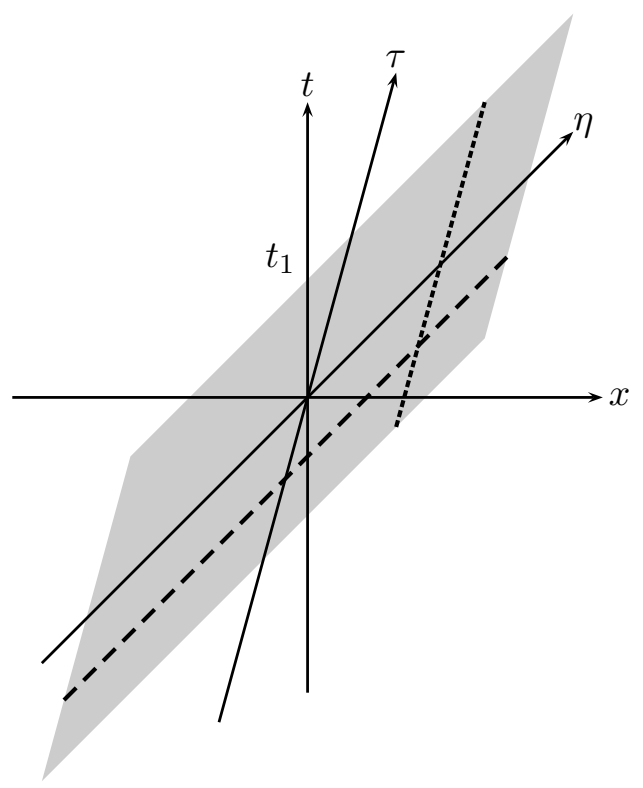

FIG. 1: The parallelogram $\Phi_{v}(\eta, \tau)$, shown shaded, can be considered as a set of timelike geodesics parameterized by $\tau$ with $\eta$ fixed (short dashes), or of null geodesics parameterized by $\eta$ with $\tau$ fixed (long dashes).

Let us consider $\gamma$ sufficiently large that $t_{1}<t_{0}$. Then, since the integral in Eq. (6) converges uniformly, we can find a number $\lambda_{1}$ such that

$$
\int_{-\eta_{0}}^{\eta_{0}} d \eta T_{\omega}\left(\Phi_{v}(\eta, \tau)\right)=\int_{\lambda_{-}}^{\lambda_{+}} d \lambda T_{\omega}\left(\Phi_{0}(\lambda, t)\right)<-A / 2
$$

for all $t \in\left(-t_{1}, t_{1}\right)$, as long as

$$
\begin{aligned}
& \lambda_{+}>\lambda_{1} \\
& \lambda_{-}<-\lambda_{1} .
\end{aligned}
$$

By integrating Eq. (16), we find

$$
\int_{-\tau_{0}}^{\tau_{0}} d \tau \int_{-\eta_{0}}^{\eta_{0}} d \eta T_{\omega}\left(\Phi_{v}(\eta, \tau)\right) f\left(\tau / \tau_{0}\right)^{2}<-\frac{A}{2} \int_{-\tau_{0}}^{\tau_{0}} d \tau f\left(\tau / \tau_{0}\right)^{2}=-\frac{A \tau_{0}}{2}
$$

Now choose any fixed, positive $\tau_{0}<r$. Let $\gamma$ grow without bound and let $\eta_{0}=\lambda_{1}+v \gamma \tau_{0}$ to satisfy Eqs. (17). Then the right hand side of Eq. (13) goes as $\gamma^{-1}$, while that of Eq. (18) is constant, so the two inequalities cannot simultaneously be satisfied in this limit. This contradiction completes the proof of Theorem II.1.

Another consequence of our result in this section is that if the state $\omega$ is sufficiently regular that the integral in Eq. (6) converges uniformly for all $t$ in a neighborhood of $t=0$ contained in $(-r, r)$, then we have $A(t) \geq 0$ for all such $t$ : this follows by applying Theorem II.1 to the translates $\lambda \mapsto \Phi_{0}(\lambda, t)$ of $\zeta$, which also satisfy the geometrical conditions of Sec. IIA (albeit with possibly smaller tubular neighborhoods $N_{r}^{\prime}$ ).

\section{A VARIANT OF THE ARGUMENT}

In Sec. [II we showed that no Hadamard state can violate the ANEC uniformly on a timelike family of geodesics, where violation of ANEC was interpreted to mean that the 
ANEC integral converges to a negative value. It is also of interest to consider weaker versions of ANEC (as in [13]) in which convergence of the integral is not required. The spacetime $(N, \boldsymbol{g})$, null geodesic $\zeta$ and neighborhood $N^{\prime}$ are assumed to obey the conditions of Secs. ПA, ПB, Our result is the following.

Theorem III.1 Suppose that $T_{\omega}$ is uniformly continuous in $N^{\prime}$ for some Hadamard state $\omega$. Then

$$
\liminf _{\lambda_{0} \rightarrow \infty} \int d \lambda T_{\omega}(\zeta(\lambda)) f\left(\lambda / \lambda_{0}\right)^{2} \geq 0
$$

where $f$ is any smooth real-valued function supported in the interval $(-1,1)$.

To prove this result, we approximate the null segment $\left\{\zeta(\lambda):|\lambda| \leq \lambda_{0}\right\}$, which contains the support of the integrand, by a timelike segment $\Phi_{v}(0, \tau)$ [as defined in Eq. (7)] with velocity $v$ and boost $\gamma$, and the correspondence $\tau=\lambda / \gamma$. The velocity should be large enough that $\lambda_{0} / \gamma<r$ in order to apply the Minkowski space QNEI along the timelike curve. The distance between corresponding points is

$$
\left|\zeta(\lambda)-\Phi_{v}(0, \tau)\right|=\gamma(1-v)|\tau|<|\tau| / \gamma<\lambda_{0} / \gamma^{2}
$$

and thus by uniform continuity,

$$
\left|T_{\omega}(\zeta(\lambda))-T_{\omega}\left(\Phi_{v}(0, \tau)\right)\right|<C \lambda_{0} / \gamma^{2}
$$

where $C$ is a positive constant. Integration gives

$$
\int d \lambda T_{\omega}(\zeta(\lambda)) f\left(\lambda / \lambda_{0}\right)^{2}-\int d \lambda T_{\omega}\left(\Phi_{v}(0, \tau)\right) f\left(\tau / \tau_{0}\right)^{2}>-2 C \lambda_{0}^{2} / \gamma^{2}
$$

with $\tau=\lambda / \gamma$. Changing variables in the second integral and using Eq. (11) gives

$$
\int d \lambda T_{\omega}(\zeta(\lambda)) f\left(\lambda / \lambda_{0}\right)^{2}>-\frac{F}{12 \pi^{2} \gamma \tau_{0}^{3}}-\frac{2 C \lambda_{0}^{2}}{\gamma^{2}}=-\frac{F \gamma^{2}}{12 \pi^{2} \lambda_{0}^{3}}-\frac{2 C \lambda_{0}^{2}}{\gamma^{2}}
$$

Now let $\lambda_{0}$ grow without bound, and let $v$ change so that $\gamma=\lambda_{0}^{5 / 4}$ (thus $\lambda_{0} / \gamma \rightarrow 0$ ). The right hand side of Eq. (23) goes to zero, and we conclude that Eq. (19) holds, which is the form in which the ANEC was formulated in [13]. This completes the proof of Theorem III.1.

A number of other hypotheses also lead to the conclusion Eq. (19). Noting that the difference between the points in Eq. (21) is purely in the $x$ direction, it is sufficient to have $\partial T_{\omega} / \partial x$ bounded in place of uniform continuity. Similarly, we could modify the proof so that the timelike and null curves are related by the correspondence $\tau=\lambda /(v \gamma)$, in which case the corresponding points differ only in $t$, and it is sufficient to have $\partial T_{\omega} / \partial t$ bounded. In particular, we can prove Eq. (19) in this way for any stationary configuration of fields. Note also that the conclusion of Theorem III.1 applies equally to any other complete null geodesic contained in $N_{r}^{\prime}$.

\section{EXAMPLE: THE CYLINDER SPACETIME}

One of the simplest examples in which the ANEC is known to be violated is the cylinder spacetime obtained by periodically identifying Minkowski space along the $z$-direction. The 
ground state of the massless, minimally coupled scalar field on this (globally hyperbolic) spacetime has renormalized stress tensor

$$
\left\langle T_{a b}\right\rangle=\frac{\pi^{2}}{90 L^{4}} \operatorname{diag}(-1,1,1,-3)
$$

where $L$ is the periodicity length. It is readily verified that the (A)NEC is violated along any null geodesic with a nonzero $z$-component of velocity, i.e., for those which wind around the compact direction. On the other hand, the (A)NEC is obeyed along complete null geodesics with zero velocity in the $z$-direction. We wish to show how this follows from our main results.

First, note that null geodesics which wind round the cylinder are not achronal, and are therefore excluded by our hypotheses. So the violation of the ANEC along these curves is not in contradiction with our results. Now consider any complete null geodesic $\zeta$ with zero velocity in the $z$-direction. Without loss of generality, we may assume that its three-velocity is directed in the positive $x$-direction. We will construct a neighborhood $N^{\prime}$ of $\zeta$ that satisfies the conditions set out in Sec. IIA.

Let $M^{\prime}=\left\{(t, x, y, z) \in M:(t-x)^{2}+y^{2}+z^{2}<r^{2}\right\}$ for some $r<L / 4$. Then we define $N^{\prime}=\alpha\left(M^{\prime}\right)$, where $\alpha: M \rightarrow N$ is the quotient mapping that implements the periodic identification. Because $|z|<r<L / 2$ in $M^{\prime},(t, x, y, z)$ are good coordinates on $N^{\prime}$; moreover, $N^{\prime}$ is simply connected and does not taper off at infinity. Since the Riemann tensor vanishes, we now need only check that condition (2) holds. Let $\tau_{n}$ represent a translation through $L$ along the $z$-axis in $M$ and let $p$ be an arbitrary point of $M^{\prime}$. Then

$$
J^{+}(\alpha(p), N) \cap N^{\prime}=\alpha\left(M^{\prime} \cap \bigcup_{n \in \mathbb{Z}} J^{+}\left(\tau_{n} p, M\right)\right)
$$

while

$$
J^{+}\left(\alpha(p), N^{\prime}\right)=\alpha\left(J^{+}\left(p, M^{\prime}\right)\right)=\alpha\left(M^{\prime} \cap J^{+}(p, M)\right),
$$

where the equality $J^{+}\left(p, M^{\prime}\right)=M^{\prime} \cap J^{+}(p, M)$ holds because $M^{\prime}$ is convex. Condition (2) will follow if we can show that $J^{+}\left(\tau_{n} p, M\right) \cap M^{\prime} \subset J^{+}(p, M)$ for each $n \in \mathbb{Z}$. This could fail only if there were a point $q \in M^{\prime}$ which was spacelike separated from $p$, but timelike separated (in $M$ ) from some image point $p^{\prime}$ of $p$. But the difference in the $z$-coordinate of $p$ and $q$ must be less than $L / 2$ (as both lie in $M^{\prime}$ ) while the difference in the $z$ coordinate of $q$ and $p^{\prime}$ must be greater than $L / 2$. Thus the displacement from $p^{\prime}$ to $q$ must be 'more spacelike' than that from $p$ to $q$, and we see that no such $q$ can exist. Thus condition (2) holds, and our results do apply to $\zeta$; not only in the ground state, but in all Hadamard states on this spacetime. Moreover, they would continue to apply even if we perturbed the metric on $(N, \boldsymbol{g})$ outside $N^{\prime}$, provided the new metric respects condition (2).

\section{DISCUSSION}

We have given a proof of the ANEC for null geodesics with suitable Minkowskian tubular neighborhoods, subject to conditions which we feel are reasonable and do not impact its practical applicability. Our result is complementary to that of Flanagan and Wald [18], who found that the ANEC is enforced in semiclassical quantum gravity up to second order in perturbation theory about Minkowski spacetime, albeit with a modicum of transverse smearing on the order of a few Planck lengths. 
The main import of the present work is that distant interactions or boundary conditions apparently do not help to produce ANEC violations. For example, Casimir plates would not seem to be useful to support a wormhole, as in [2], because of the need to get through the plate [27].

This paper is the first step in a program of proving that wormholes, time machines and other such interesting phenomena are not allowed within the domain of validity of the semiclassical Einstein equation. ("Self-supporting" systems in which the geometry itself will induce the ANEC violation necessary to produce it are known [19, 20, 21]. However, they have consistently been shown to occur only on the order of the Planck scale where the semiclassical approach is itself suspect, and we must await a full quantum theory of gravity.) We have handled the case of free fields in flat space with boundary conditions. We hope in future work to be able to address interacting fields and curved spacetimes.

\section{Acknowledgments}

The authors would like to thank Larry Ford and Noah Graham for helpful discussions. K. D. O. was supported in part by the National Science Foundation under grant 0353314 and by grant RFP1-06-024 from The Foundational Questions Institute (fqxi.org) . M. J. P. would like to acknowledge a grant for support of this work from the US Army Research Office through the USMA Photonics Research Center.

[1] K. D. Olum, Phys. Rev. Lett. 81, 3567 (1998), gr-qc/9805003.

[2] M. S. Morris, K. S. Thorne, and U. Yurtsever, Phys. Rev. Lett. 61, 1446 (1988).

[3] S. W. Hawking, Phys. Rev. D46, 603 (1992).

[4] F. J. Tipler, Annals Phys. 108, 1 (1977).

[5] H. B. G. Casimir, Kon. Ned. Akad. Wetensch. Proc. 51, 793 (1948).

[6] G. J. Galloway, Manuscr. Math. 35, 209 (1981).

[7] T. A. Roman, Phys. Rev. D33, 3526 (1986).

[8] T. A. Roman, Phys. Rev. D37, 546 (1988).

[9] G. Klinkhammer, Phys. Rev. D43, 2542 (1991).

[10] A. Folacci, Phys. Rev. D46, 2726 (1992).

[11] L. H. Ford and T. A. Roman, Phys. Rev. D51, 4277 (1995), gr-qc/9410043.

[12] C. J. Fewster and T. A. Roman, Phys. Rev. D67, 044003 (2003), gr-qc/0209036.

[13] R. Wald and U. Yurtsever, Phys. Rev. D44, 403 (1991).

[14] R. Verch, J. Math. Phys. 41, 206 (2000), math-ph/9904036.

[15] C. J. Fewster and S. Hollands, Rev. Math. Phys. 17, 577 (2005), math-ph/0412028.

[16] U. Yurtsever, Phys. Rev. D51, 5797 (1995), gr-qc/9411056.

[17] M. Visser, Phys. Rev. D54, 5116 (1996), gr-qc/9604008.

[18] E. E. Flanagan and R. M. Wald, Phys. Rev. D54, 6233 (1996), gr-qc/9602052.

[19] R. Garattini, Class. Quant. Grav. 22, 1105 (2005), gr-qc/0501105.

[20] D. Hochberg, A. Popov, and S. V. Sushkov, Phys. Rev. Lett. 78, 2050 (1997), gr-qc/9701064.

[21] N. R. Khusnutdinov and S. V. Sushkov, Phys. Rev. D65, 084028 (2002), hep-th/0202068.

[22] M. J. Pfenning and L. H. Ford, Class. Quant. Grav. 14, 1743 (1997), gr-qc/9702026.

[23] L. H. Ford and T. A. Roman, Phys. Rev. D53, 5496 (1996), gr-qc/9510071. 
[24] K. D. Olum and N. Graham, Phys. Lett. B554, 175 (2003), gr-qc/0205134.

[25] D. Schwartz-Perlov and K. D. Olum, Phys. Rev. D68, 065016 (2003), hep-th/0307067.

[26] N. Graham, K. D. Olum, and D. Schwartz-Perlov, Phys. Rev. D70, 105019 (2004), grqc/0407006.

[27] N. Graham and K. D. Olum, Phys. Rev. D72, 025013 (2005), hep-th/0506136.

[28] D. Schwartz-Perlov and K. D. Olum, Phys. Rev. D72, 065013 (2005), hep-th/0507013.

[29] C. J. Fewster and M. J. Pfenning, J. Math. Phys. 47, 082303 (2006), math-ph/0602042.

[30] R. Brunetti, K. Fredenhagen, and R. Verch, Commun. Math. Phys. 237, 31 (2003), math$\mathrm{ph} / 0112041$.

[31] S. W. Hawking and G. F. R. Ellis, The Large Scale Structure of Space-time (Cambridge University Press, London, 1973).

[32] C. J. Fewster and M. J. Pfenning, (in preparation). 